\title{
DETERMINACIÓN DE LAS PROPIEDADES TERMALES EN TRES VARIEDADES DE PAPA NATIVA CON CORTES DE TAMAÑOS DIFERENTES
}

\author{
DETERMINATION OF THE THERMAL PROPERTIES IN THREE NATIVE \\ POTATO VARIETIES WITH CUTS OF DIFFERENT SIZES
}

\author{
${ }^{1}$ David Juan Ramos Huallpartupa
}

\begin{abstract}
RESUMEN
El presente trabajo determina los valores de las propiedades termales (difusividad, conductividad y calor específico) de tres variedades de papa nativa (Imilla negra, Marihua y Gaspar) con dos tipos de cortes diferentes, para ello se recolectó tubérculos provenientes del mercado central de la ciudad de Andahuaylas, en estado de madurez comercialmente óptimo. Se generó 06 condiciones diferentes de estudio cualitativas con tres variedades y dos tipos de cortes. Se realizó la determinación de las propiedades termales utilizando equipos prediseñados para la Conductividad y la Difusividad Termal, así mismo se realizaron balances de energía y cálculos para encontrar los valores de las propiedades termales. Los resultados fueron obtenidos y analizados mediante un arreglo $3 \times 2$ con tres repeticiones, encontrándose valores que se acercan a los citados en la bibliografia, que difieren entre sí por el tipo de corte en cada variedad; también se determinó la influencia altamente significativa en la variación de las propiedades termales debida al tipo de corte.
\end{abstract}

Palabras clave: Papa nativa, conductividad termal, difusividad termal, calor específico, tipo de corte.

\section{ABSTRACT}

The values of the thermal properties (diffusivity, conductivity and specific heat) of three native potato varieties (black Imilla, Marihua and Gaspar) were determined with 02 different types of cut; to do this, tubers from the central market of Andahuaylas's city with were gathered. Six different conditions of qualitative study were generated; these are 03 varieties and 02 types of cuts. The determination of the thermal properties by using predesigned equipments for the Conductivity and the Thermal Diffusivity was done; likewise, energy balances and calculations to find the values of the thermal properties were performed. The results were obtained and analyzed by means of a $3 \times 2$ arrangement with three replications, finding values close to the ones mentioned in the bibliography and differing from each other by the type of cut in every variety; also, the highly significant influence of the variation of the thermal properties due to the type of cut.

Key words: native potato, thermal conductivity, thermal diffusivity, specific heat, type of cut.

\section{INTRODUCCIÓN}

Tomar en consideración la alimentación es indispensable para la producción agroindustrial, por ello la seguridad agroalimentaria conlleva la preservación de alimentos para épocas de escases, así como el conocimiento de las propiedades termales de los productos con fines de mejorar la potencialidad de productos procesados mediante tratamientos térmicos.

Entre los problemas que tienen los productores e industriales, respecto a la utilización integral de los productos agropecuarios, es que dejan de lado el conocimiento de los procesos de transformación y el ahorro de energía que demandan, debido al poco conocimiento de algunas propiedades involucradas en los tratamientos térmicos, provocando así pérdidas económicas al productor por el mal uso de la energía.

Los materiales cambian sus propiedades con la temperatura; en la mayoría de casos las propiedades mecánicas y físicas dependen de la temperatura a la cual se usa o somete el material durante su procesamiento. (Alvarado y Aguilera, 2001).

Las propiedades térmicas son parámetros que reflejan la capacidad de predicción de las velocidades de transferencia de calor para calentamiento o congelamiento de productos alimenticios.

Singh y Heldman (2008) mencionan que la importancia de la determinación de las propiedades térmicas en alimentos permite realizar el cálculo de transferencia de calor. El cálculo de las velocidades de transferencia de calor así como para procesos de calentamiento y enfriamiento, y para su utilización adecuada en el diseño de equipos de procesos, están basados en la ecuación de trasferencia de calor en estado transitorio:

$$
\frac{d T}{d t}=\alpha\left(\frac{d^{2} T}{d x^{2}}+\frac{d^{2} T}{d y^{2}}+\frac{d^{2} T}{d z^{2}}\right)
$$


Ramos, D., Determinación de las propiedades termales en tres variedades de papa nativa con cortes de tamaños diferentes.

Dónde: $\mathrm{T}=$ es la temperatura en cualquier punto dado por las coordenadas $\mathrm{x}, \mathrm{y}, \mathrm{z} . ; \mathrm{t}=\mathrm{es}$ el tiempo; $\alpha=$ es la difusividad termal.

El calor específico es una propiedad que e indicativo de la habilidad de un material para absorber calo de los alrededores. Esta representa la cantidad de energi: requerida para producir un aumento de la unidad di temperatura en $1{ }^{\circ} \mathrm{C}$. (Mohsenin, 1980).

La conducción térmica es el fenómeno por medic del cual el calor se transporta de una región de alt: temperatura a una de baja temperatura. Es la propiedad qui caracteriza la habilidad de un material de transferir calor. L: cual se define como la gradiente de temperatura entre do superficies hacia el cual fluye el calor, la cantidad de calor qui fluye en una unidad de tiempo, a través del área, se llam: conductividad térmica. (Mohsenin, 1980).

La difusividad térmica es una propiedac importante para la simulación de procesos en que 1 : transmisión de calor se efectúa en estado transitorio. E significado físico de la difusividad térmica consiste et determinar lo rápido que el calor se propaga o difunde : través de un material, es decir la rapidez con la que si propaga la temperatura cuando hay calentamiento c enfriamiento. Esta propiedad también es definida como $\mathrm{l}$ : relación entre tres propiedades diferentes de acuerdo con 1 : siguiente ecuación (Alvarado, 2001).

$$
\alpha=\frac{k}{\rho C_{p}}
$$

Dónde: $\alpha=$ difusividad térmica; $k=$ conductividac térmica; $\rho=$ densidad; $C_{p}=$ Calor especifico del alimento.

El conocimiento de las propiedades termo físicas de los alimentos sólidos y líquidos en un amplio rango de concentraciones y temperaturas es de gran importancia en la industria, ejemplo: alimentos concentrados, secados, esterilizados, refrigerados o congelados, ya que son vitales en operaciones unitarias como bombeo, intercambio de calor, evaporación, congelamiento y secado, las cuales deben ser correctamente proyectadas y controladas.

Las propiedades termales de los productos vegetales son atribuidas principalmente al valor de la difusividad termal, la conductividad termal así como el calor especifico, todas variables en función al contenido de nutrientes considerados como sólidos totales presentes en las diferentes variedades de papa, por lo que es importante conocer las propiedades termales, esto con la finalidad de realizar operaciones y procesos de tratamiento térmico en las diferentes variedades de papa y cortes, planteadas en el presente trabajo.

\section{MATERIALES Y MÉTODOS}

\section{Materiales}

Se utilizó 03 variedades de papa nativa (imilla negra, marihua, gaspar); materiales de laboratorio, termómetros digitales, equipo para determinación de difusividad termal, equipo para determinar conductividad termal, termocuplas, baño maría termostatizada, DATA TRACE, transformador de voltaje.

\section{Metodología del trabajo experimental:}

El trabajo de investigación se desarrolló paso a naen mediante el cimiente diceño evnerimental (Fimura 01 ).

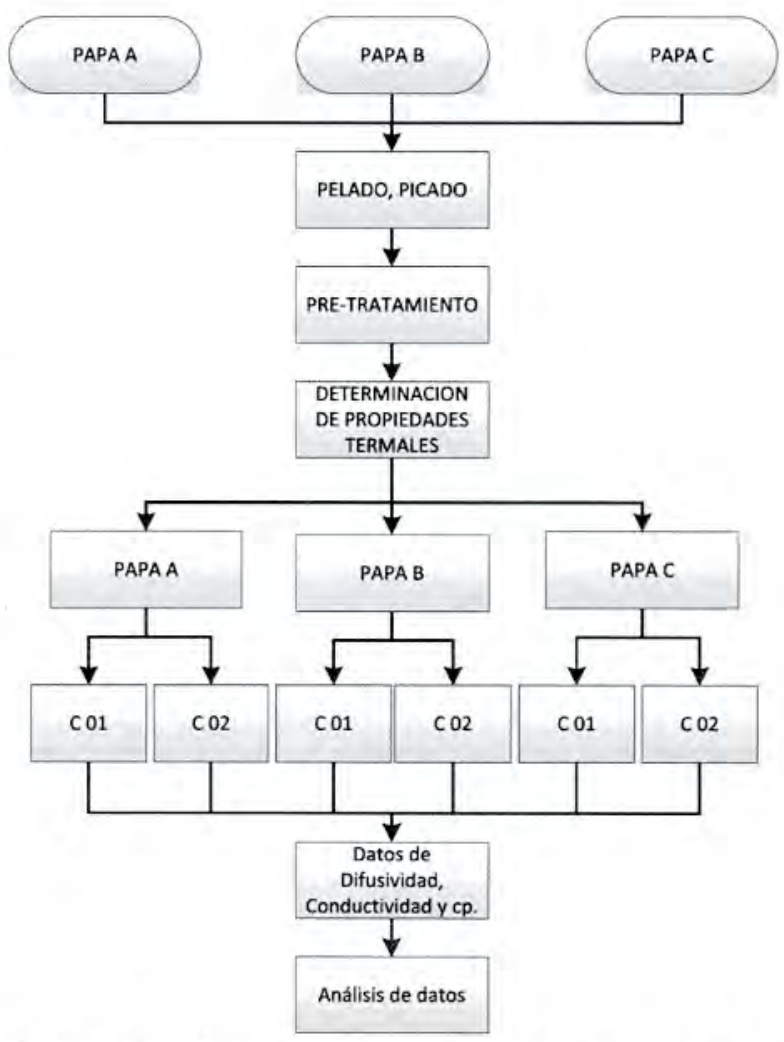

Figura 01. Metodología experimental con el que se determinó los valores de las propiedades termales FUENTE: Elaboración propia

Leyenda:

Papa A: Imilla negra. C01: Corte en láminas

Papa B: Marihua. $\quad$ C02: Corte en cubos $\left(0,125 \mathrm{~cm}^{3}\right)$

Papa C: Gaspar.

Para evaluar y determinar la implicancia de los factores en estudio, los datos fueron distribuidos bajo un DBCA y analizados bajo un arreglo $3 \times 2 \times 3$ obteniéndose 06 tratamientos con 18 observaciones, los datos fueron sometidos a un análisis de varianza al $95 \%$ de probabilidades; los estudios se complementaron con pruebas de comparación múltiple a fin de ordenar las variedades de papa en función a las propiedades termales.

\section{Metodología para los análisis}

Para determinar la difusividad termal en las 03 variedades de papa con cortes diferentes, se construyó un equipo experimental basado en la metodología modificada por Ureña (1990), partiendo de la metodología de Dickerson (1965) y realizando un registro de datos del tiempo y temperatura mediante el DATA TRACE hasta la proximidad de temperatura de equilibrio, para luego llevarla al cálculo de la difusividad termal mediante la ecuación desarrollada:

$$
t=0,398 \frac{r^{2}}{\alpha} \log \left(1,6 \frac{T_{a}-T_{0}}{T_{a}-T}\right)
$$

Tiempo (t); radio del cilindro (r); Temperatura del medio 
$\left(\mathrm{T}_{2}\right)$; Temperatura inicial $\left(\mathrm{T}_{\mathrm{o}}\right)$; Temperatura deseada $(\mathrm{T})$; Difusividad termal $(\alpha)$.

Para determinar el calor específico de las 03 variedades de papa con cortes diferentes, se realizó un balance de energía en el sistema del equipo experimental, considerando:

$$
Q_{s}=Q_{\mathrm{A}}+\mathrm{Q}_{\mathrm{D}}
$$

Calor suministrado $\left(\mathrm{Q}_{2}\right)$; Calor absorbido $\left(\mathrm{Q}_{N}\right)$; Calor disipado $\left(\mathrm{Q}_{12}\right)$

Para determinar la conductividad termal en las 03 variedades de papa con cortes diferentes, además de la obtención de la densidad del producto, se realizó un cálculo mediante la siguiente relación (Singh, 2008):

$$
\alpha=\frac{k}{\rho C_{p}}
$$

Conductividad termal $(k)$; densidad del producto $(\rho)$; calor específico $\left(C_{n}\right)$

\section{RESULTADOS}

Determinación de los valores de las propiedades termales (difusividad, conductividad y calor específico) de tres variedades de papa nativa con cortes diferentes.

En las tablas 1,2 y 3 se muestran los resultados de los valores de la propiedades termales obtenidos mediante la determinación en laboratorio y siguiendo estrictamente la metodología planteada.
Evaluación de los valores de las propiedades termales determinadas para cada variedad de papa con cortes diferentes.

La evaluación de los valores de las propiedades termales se realizaron mediante un análisis de varianza, lográndose observar que existe diferencias estadísticas altamente significativas $(\mathrm{p}<=0,05)$ para los efectos principales de los factores (variedad de papa nativa y tipo de corte) en estudio; así mismo existe diferencia altamente significativa para la interacción entre variedad de papa nativa y tipo de corte, lo que nos afirma que estos dos factores son dependientes, es decir que para cierto nivel de variedad hay que estudiar cual es la mejor propiedad termal, anulando asi los efectos principales de los factores variedad de papa nativa y tipo de corte, y adquieren mayor importancia los efectos simples para nuestras conclusiones. Cada evaluación se muestra en las tablas 4,5 y 6 , que corresponde al análisis de varianza para cada propiedad termal.

Determinación de la influencia de los cortes realizados en la determinación del valor de las propiedades termales.

Del análisis estadístico realizado, la influencia de los cortes en las propiedades termales es altamente significativa, obteniéndose en promedio los resultados mostrados en la Tabla 7.

\begin{tabular}{|c|c|c|c|c|c|c|}
\hline \multirow[t]{2}{*}{ REP } & \multicolumn{2}{|c|}{ PAPA “A" } & \multicolumn{2}{|c|}{ PAPA "B" } & \multicolumn{2}{|c|}{ PAPA “C” } \\
\hline & C 01 & C 02 & C 01 & C 02 & C 01 & C 02 \\
\hline 1 & $4,8843 \mathrm{E}-08$ & $5,1888 \mathrm{E}-08$ & $5,7610 \mathrm{E}-08$ & $4,3646 \mathrm{E}-08$ & $5,8753 \mathrm{E}-08$ & $5,8665 \mathrm{E}-08$ \\
\hline 2 & $4,7946 \mathrm{E}-08$ & $5,0894 \mathrm{E}-08$ & $5,6990 \mathrm{E}-08$ & $4,3557 \mathrm{E}-08$ & $5,8700 \mathrm{E}-08$ & $5,8612 \mathrm{E}-08$ \\
\hline 3 & $4,9002 \mathrm{E}-08$ & $5,1932 \mathrm{E}-08$ & $5,7702 \mathrm{E}-08$ & $4,3646 \mathrm{E}-08$ & $5,8761 \mathrm{E}-08$ & $5,8712 \mathrm{E}-08$ \\
\hline \multicolumn{7}{|c|}{ Tabla $\mathrm{N}^{\circ} 02$. Resultados de conductividad termal $\left(\mathrm{W} / \mathrm{m}^{\circ} \mathrm{C}\right)$} \\
\hline & \multicolumn{2}{|c|}{ PAPA “A" } & \multicolumn{2}{|c|}{ PAPA “B” } & \multicolumn{2}{|c|}{ PAPA “C" } \\
\hline REP & C 01 & C 02 & C 01 & C 02 & C 01 & $\mathrm{C} 02$ \\
\hline 1 & 0,2399 & 0,2262 & 0,2919 & 0,1759 & 0,3245 & 0,2334 \\
\hline 2 & 0,2588 & 0,2311 & 0,3001 & 0,1902 & 0,3312 & 0,2410 \\
\hline 3 & 0,2412 & 0,2299 & 0,2987 & 0,1846 & 0,3267 & 0,2357 \\
\hline \multicolumn{7}{|c|}{ Tabla $N^{\circ}$ 03. Resultados de calor especifico $\left(\mathrm{KJ} / \mathrm{Kg}^{\circ} \mathrm{C}\right)$} \\
\hline REP & \multicolumn{2}{|c|}{ PAPA “A” } & \multicolumn{2}{|c|}{ PAPA "B" } & \multicolumn{2}{|c|}{ PAPA“C” } \\
\hline & C 01 & $\mathrm{C} 02$ & C 01 & $\mathrm{C} 02$ & C 01 & C 02 \\
\hline 1 & 4,9892 & 4,8078 & 5,1468 & 4,5073 & 5,5371 & 4,5160 \\
\hline 2 & 4,9896 & 4,8069 & 5,1033 & 4,5112 & 5,5124 & 4,5032 \\
\hline 3 & 4,9800 & 4,8100 & 5,1366 & 4,5021 & 5,5484 & 4,5158 \\
\hline
\end{tabular}

Tabla $\mathbf{N}^{\circ} 01$. Resultados de difusividad termal $(\mathrm{m} 2 / \mathrm{s})$

Tabla $\mathbf{N}^{\circ} 04$. Análisis de varianza para la difusividad termal

\begin{tabular}{cccccc} 
F de V & G.L. & S.C. & C.M. & Fc. & N.S. \\
\hline Variedad & 2 & $2,82535 \mathrm{E}-16$ & $1,41267 \mathrm{E}-16$ & 1027,15469 & $* *$ \\
Corte & 1 & $5,95941 \mathrm{E}-17$ & $5,95941 \mathrm{E}-17$ & 433,308475 & $* *$ \\
$\mathrm{~V}^{*} \mathrm{C}$ & 2 & $2,40074 \mathrm{E}-16$ & $1,20037 \mathrm{E}-16$ & 872,788732 & $* *$ \\
Error Exp & 12 & $1,6504 \mathrm{E}-18$ & $1,3753 \mathrm{E}-19$ & & \\
& 17 & $5,83854 \mathrm{E}-16$ & & & \\
CV & 0,698 & $\%$ & & & \\
\hline
\end{tabular}


Ramos, D., Determinación de las propiedades termales en tres variedades de papa nativa con cortes de tamaños diferentes.

Tabla $\mathbf{N}^{\circ} \mathbf{0 5}$. Análisis de varianza para la conductividad termal

\begin{tabular}{|c|c|c|c|c|c|}
\hline $\mathbf{F}$ de V & G.L. & S.C. & C.M. & Fc. & N.S. \\
\hline Variedad & 2 & 0,00742194 & 0,00371097 & 103,013666 & $* *$ \\
\hline Corte & 1 & 0,02457445 & 0,02457445 & 682,167983 & ** \\
\hline $\mathrm{V}^{*} \mathrm{C}$ & 2 & 0,00752503 & 0,00376252 & 104,444583 & ** \\
\hline \multirow[t]{2}{*}{ Error Exp } & 12 & $4,3229 \mathrm{E}-04$ & $3,6024 \mathrm{E}-05$ & & \\
\hline & 17 & 0,03995371 & & & \\
\hline $\mathrm{CV}$ & 2,369 & $\%$ & & & \\
\hline \multicolumn{6}{|c|}{ Tabla $\mathrm{N}^{\circ} 06$. Análisis de varianza para el calor especifico } \\
\hline$F$ de V & G.L. & S.C. & C.M. & Fc. & N.S. \\
\hline Variedad & 2 & 0,12722471 & 0,06361235 & 396,028331 & $* *$ \\
\hline Corte & 1 & 1,65794991 & 1,65794991 & 10321,8179 & $* *$ \\
\hline $\mathrm{V}^{*} \mathrm{C}$ & 2 & 0,53340887 & 0,26670443 & 1660,40878 & ** \\
\hline \multirow[t]{2}{*}{ Error Exp } & 12 & $1,9275 \mathrm{E}-03$ & $1,6063 \mathrm{E}-04$ & & \\
\hline & 17 & 2,32051099 & & & \\
\hline $\mathrm{CV}$ & 0,258 & $\%$ & & & \\
\hline
\end{tabular}

Tabla $N^{\circ}$ 07. Resultados de cada propiedad termal en función al tipo de corte en cada variedad de papa

\begin{tabular}{ccccccc}
\hline Propiedad Termal & \multicolumn{2}{c}{ PAPA “A” } & \multicolumn{2}{c}{ PAPA "B" } & \multicolumn{2}{c}{ PAPA “C” } \\
& C 01 & C 02 & C 01 & C 02 & C 01 & C 02 \\
\hline Difusividad $\left(\mathrm{m}^{2} / \mathrm{s}\right)$ & $4,860 \mathrm{E}-08$ & $5,157 \mathrm{E}-08$ & $5,743 \mathrm{E}-08$ & $4,362 \mathrm{E}-08$ & $5,874 \mathrm{E}-08$ & $5,866 \mathrm{E}-08$ \\
Conductividad $\left(\mathrm{W} / \mathrm{m}^{\circ} \mathrm{C}\right)$ & 0,2466 & 0,2291 & 0,2969 & 0,1836 & 0,3275 & 0,2367 \\
Calor especifico $\left(\mathrm{KJ} / \mathrm{Kg}^{\circ} \mathrm{C}\right)$ & 4,9863 & 4,8082 & 5,1289 & 4,5069 & 5,5326 & 4,5117 \\
\hline
\end{tabular}

\section{DISCUSIÓN}

Los valores de las propiedades termales (difusividad, conductividad y calor específico) de tres variedades de papa nativa con cortes diferentes, se muestran en la Tabla 7. Singh y Erdogdu (2004) muestra valores de: difusividad termal igual a $1,7 \times 10^{8} \mathrm{~m}^{2} / \mathrm{s}$; conductividad termal $0,554 \mathrm{~W} / \mathrm{m}^{\circ} \mathrm{C}$, calor especifico de $3,52 \mathrm{KJ} / \mathrm{Kg}^{\circ} \mathrm{C}$. Al realizar una comparación, los valores obtenidos en la investigación se acercan a lo reportado por Singh y Erdogdu (2004), Geankoplis (1998), Ibarz y Barbosa (2003) y Singh (1982).

Esta cercanía de los valores es debido a la variedad de papa que se está investigando, del cual podemos afirmar que hay diferencia entre los tipos de cortes, puesto que el flujo de calor es mucho mayor en aquel que tiene dimensiones más pequeñas. Esto se explica con los valores de las propiedades termales obtenidas.

De las 06 muestras, la papa nativa Marihua con corte de cubos tiene $4,362 \mathrm{E}-08 \mathrm{~m}^{2} / \mathrm{s}$ de difusividad termal; $0,1836 \mathrm{~W} / \mathrm{m}^{\circ} \mathrm{C}$ de conductividad termal y $4,5069 \mathrm{KJ} / \mathrm{Kg}^{\circ} \mathrm{C}$ de calor especifico, lo que nos indica que con el corte en cubos de $0,0125 \mathrm{~m} 3$, deja pasar en forma "rápida" el calor, es decir que se puede hacer procesos de cocción en tiempos cortos.

La papa nativa de variedad Gaspar presenta el mayor valor en difusividad termal, lo que implica que la velocidad de calor se difunde muy fácilmente y no así su conductividad térmica.

Los calores específicos en general muestran valores que nos indican el requerimiento de energía necesaria para elevar un grado Celsius en 1 kilogramo de papa. La que requiere más energía es la variedad Gaspar con corte de láminas. Así mismo esta variedad presenta un valor alto en su conductividad, lo que implica que contiene alto contenido de sólidos que no permiten fácilmente el paso de energía.

Al evaluar los valores de las propiedades termales determinadas para cada variedad de papa con cortes diferentes podemos afirmar que existe diferencias estadísticas altamente significativas $(\mathrm{p}<=0,05)$ para los efectos principales de los factores (variedad de papa nativa y tipo de corte) en estudio; así mismo existe diferencia altamente significativa para la interacción variedad de papa nativa y tipo de corte, lo que nos afirma que estos dos factores son dependientes, es decir que para cierto nivel de variedad hay que estudiar cual es la mejor propiedad termal, anulando así los efectos principales de los factores variedad de papa nativa y tipo de corte, y adquiere mayor importancia los efectos simples.

Del análisis estadístico realizado se desprende que la influencia de los cortes en las propiedades termales es altamente significativa.

\section{CONCLUSIONES}

- Existe diferencias estadísticas altamente significativas $(\mathrm{p}<=0,05)$ para los efectos principales de los factores (variedad de papa nativa y tipo de corte) en estudio; así mismo existe diferencia altamente significativa para la interacción variedad de papa nativa y tipo de corte, lo que confirma que estos dos factores son dependientes, es decir que para cierto nivel de variedad hay que estudiar cual es la mejor propiedad termal, anulando así los efectos principales de los factores variedad de papa nativa y tipo de corte, y adquiere mayor importancia los 
Ramos, D., Determinación de las propiedades termales en tres variedades de papa nativa con cortes de tamaños diferentes.

efectos simples.

- La influencia de los cortes en las propiedades termales es altamente significativa.

\section{RECOMENDACIONES}

- Realizar pruebas con determinación de humedad para cada muestra.

- Realizar estudios de tratamiento térmico utilizando los datos obtenidos.

\section{REFERENCIAS BIBLIOGRÁFICAS}

Alvarado, J. y Aguilera, J.M. (2001). Métodos para Medir las Propiedades Físicas en la Industria de los Alimentos, Editorial Acribia, S.A.

Dickerson, W. R. 1965. An Aparatus for the Measurement of Thermal Diffusivity of foods. Journal Food Technology, 18 (5), 198-204.

Díaz, P. B. (1994). Transferencia de calory aplicaciones. 2 da. Ed. Lima-Perú.

Dutta, S. K. (1988). Thermal properties of Gram. Journal Agri. Eng. Res. 39: 269-275.
Earle, R.L (1998). Ingenieria de Alimentos. Editorial Acribia, Zaragoza-España.

Geankoplis, C. J. (1998). Procesos de transporte y operaciones unitarias. $2 \mathrm{da}$. Ed. CECSA. México.

Ibarz, A. y Barbosa, G. (2003). Unit operations in food engineering. Inc. Madrid - España.

Lewis, M. J. (1993). Propiedades fisicas de los alimentos y de los sistemas de procesado. Editorial Acribia, Zaragoza España.

Mohsenin, N. (1980). Thermal Properties of Food and Agriculturalmaterials. Gordon and Breach Science publishers, INC. N.Y. USA. pp407.

Singh, R. P. (1982). Termal diffusivity in food processing. JournalFood Technology, 36 (2): 87-91.

Singh, P. y Heldman, R. (2008). Introducción a la Ingeniería de los Alimentos. 2da. Ed. Zaragoza - España.

Singh, R.P. y F. Erdogdu (2004); Virtual experiments in food processing, RAR Press. Davis, California.

Ureña, M. O. (1990). Obtención de Alimentos Modelo a partir de Mezclas de bentonita: Glicerol: Agua para Estudios de Tratamientos Térmicos. Valencia, España, Universidad Politécnica de Valencia, Departamento de Tecnología de Alimentos, $145 \mathrm{p}$.
Correspondencia:

David Juan Ramos Huallpartupa: davisrh@gmail.com
Fecha de Recepción: 23/10/2014

Fecha de Aceptación: 01/12/2014 